\title{
ON THE BOUNDARY CONTROL OF BEAM EQUATION
}

\author{
Ömer Morgül \\ Bilkent University, Dept. of Electrical and Electronics \\ Engineering, 06533, Bilkent, Ankara, Turkey
}

\begin{abstract}
A flexible system described by Euler-Bernoulli beam equation is considered. The beam is clamped at one end, and is free at the other end. Boundary control force and torque inputs are applied at the free end of the beam. The transfer functions of the controllers are marginally stable and may contain some poles on the imaginary axis. Various stability results are shown and the application of the proposed control law to disturbance rejection problem is considered. Copyright (C) 2002 IFAC
\end{abstract}

Keywords: Flexible beam, distributed parameter systems, stability.

\section{INTRODUCTION}

The progress in robotics and space technology has resulted in the use of lightweight materials in constructions of such systems, for various reasons including the convenience in transportation. Another reason is the need for fast rotating systems. Such mechanical systems contain parts which can be modeled as flexible structures. To achieve high precision demands for such systems, one has to take the effect of flexibility into account in designing the controllers.

Consider a mechanical system which has coupled flexible and rigid parts, such as a robot arm with a flexible link or a spacecraft with flexible appendages. The equations of motion for such systems are generally a set of coupled partial and ordinary differential equations with appropriate boundary conditions. Once the equations of motion for such systems are obtained, the commonly used approach is to express the solutions as an infinite sum in terms of the eigenfunctions corresponding to the relevant partial differential equation, and then to consider only finitely many terms in this sum, see e.g. Meirovitch (1967). This approach is called "modal" analysis and reduces the original set of equations, to a finite, although often very large, set of coupled ordinary differential equations. However, having established a control law for this reduced set of equations does not always guarantee that the same law will work on the original set of equations, (e.g., one might encounter the so-called "spillover" problems, Balas (1978)). Also note that the actual number of modes of an elastic system, in theory, is infinite and the number of modes that should be retained is not known a priori.

In recent years, the boundary control of flexible systems, (i.e., controls applied to the boundaries of the flexible parts as opposed to the controls distributed over the flexible parts), has become an important research area. This idea was first applied to the systems described by wave equation, (e.g., strings), Chen (1979), and was extented to the Euler-Bernoulli beam equation, Chen et. al. (1987). In particular, in Chen et. al. (1987), it has been proven that, in a cantilever beam, a single actuator applied at the free end of the beam is sufficient to uniformly stabilize the beam deflections. Recently, the boundary control techniques has been applied to the stabilization of a flexible spacecraft performing planar motion, Morgül (1991), and three dimensional motion Morgül (1990). For more references and technical information on this subject, the reader is referred to Luo et. al. (1999).

In this note, we consider a linear time invariant system which is represented by one-dimensional EulerBernoulli beam equation in a bounded domain. We assume that the system is clamped at one end and 
the boundary control inputs (force and torque) are applied at the other end. For this system, we propose finite dimensional dynamic boundary controllers, which generate these inputs. This introduces extra degrees of freedom in designing controllers which could be exploited in solving a variety of control problems, such as disturbance rejection, pole assignment, etc., while maintaining stability. The transfer functions of the controllers are proper rational functions of the complex variable $s$, and may contain some poles on the imaginary axis, provided that the residues corresponding to these poles are nonnegative; the rest of the transfer functions are required to be strictly positive real. This type of controllers have been proposed before for the stabilization of flexible structures, see Morgül (1994) for the wave equation, Morgül (1998) for disturbance rejection, and Morgül (1992) for the beam equation (except for the poles on the imaginary axis). We then show that if the poles on the imaginary axis do not belong to a countable set (e.g. the zeroes of a transcendence function), then the closed loop system is asymptotically stable. We also consider the case where the outputs of the controllers are corrupted by disturbance. We show that if the structure of the disturbance is known (i.e. the frequency spectrum), then it may be possible to choose the controller accordingly to attenuate the effect of the disturbance at the system output.

This paper is organized as follows. In the next section we introduce the system considered and propose a class of controllers for stabilization. In section 3 we give some stability results. In section 4 we consider disturbance rejection problem and finally we give some concluding remarks.

\section{PROBLEM STATEMENT}

We consider a flexible beam clamped at one end and is free at the other end. Without loss of generality, we assume that the beam length, mass density and the flexural rigidity are given as $L=1, \rho=1$ and $E I=1$, respectively. We denote the displacement of the beam by $u(x, t)$ at $x \in(0,1)$ and $t \geq 0$. The beam is clamped at one end and is controlled by a boundary control force at the other end. The equations are given as $(x \in(0,1), t \geq 0)$ :

$$
\begin{gathered}
u_{t t}+u_{x x x x}=0, \\
u(0, t)=0, u_{x}(0, t)=0 \\
-u_{x x}(1, t)=\alpha_{1} f_{1}(t), u_{x x x}(1, t)=\alpha_{2} f_{2}(t)
\end{gathered}
$$

where a subscript, as in $u_{t}$ denotes a partial differential with respect to the corresponding variable, and $f_{1}(\cdot), f_{2}(\cdot): \mathbf{R}_{+} \rightarrow \mathbf{R}$ are the boundary control torque and force applied at the free end of the beam, respectively. Here $\alpha_{i} \in\{0,1\}$, and $\alpha_{i}=0$ means that the corresponding controller is not applied, whereas $\alpha_{i}=1$ means that the corresponding controller is applied.
We assume that $f_{i}(t), i=1,2$, is generated by the following controller :

$$
\begin{gathered}
\dot{z}_{i}=A_{i} z_{i}+b_{i} r_{i}(t), \\
\dot{x}_{i 1}=\omega_{i} x_{i 2}, \dot{x}_{i 2}=-\omega_{i} x_{i 1}+r_{i}(t), \\
f_{i}(t)=c_{i}^{T} z_{i}+d_{i} r_{i}+k_{i} x_{i 2},
\end{gathered}
$$

where $z_{i} \in \mathbf{R}^{n_{i}}$, for some natural number $n_{i}$, is the controller state, $A_{i} \in \mathbf{R}^{n_{i} \times n_{i}}$ is a constant matrix, $b_{i}, c_{i} \in$ $\mathbf{R}^{n_{i}}$ are constant column vectors, $d_{i}, k_{i}, \omega_{i}$ are positive constants, and the superscript $T$ denotes transpose. The controller inputs $r_{i}$ are defined as :

$$
r_{1}(t)=u_{x t}(1, t) \quad, \quad r_{2}(t)=u_{t}(1, t)
$$

If we take the Laplace transform, then the controller transfer function $g_{i}(s)$ between its input $r_{i}$ and output $f_{i}(t)$ may be found as

$$
g_{i}(s)=h_{i}(s)+\frac{k_{i} s}{s^{2}+\omega_{i}^{2}},
$$

where $h_{i}(s)=c_{i}^{T}\left(s I-A_{i}\right)^{-1} b_{i}+d_{i}$.

We make the following assumptions concerning the actuator given by (4)-(6) thoroughout this work (for $i=1,2)$.

Assumption 1: All eigenvalues of $A_{i} \in \mathbf{R}^{n_{i} \times n_{i}}$ have negative real parts.

Assumption 2: $\left(A_{i}, b_{i}\right)$ is controllable and $\left(c_{i}, A_{i}\right)$ is observable.

Assumption 3 : $d_{i} \geq 0, k_{i} \geq 0$; moreover there exists a constant $\gamma_{i}, d_{i} \geq \gamma_{i} \geq 0$, such that the following holds :

$$
\mathscr{R} e\left\{h_{i}(j \omega)\right\}>\gamma_{i}, \omega \in \mathbf{R},
$$

where $h_{i}(s)$ is given in (8). Moreover for $d_{i}>0$, we assume $\gamma_{i}>0$ as well.

The assumptions given above implies that $h_{i}(s)$ is a strictly positive real transfer function, and hence $g_{i}(s)$ given by (8) is only positive real. The dynamic controller given by (8) with $\alpha_{1}=0, \alpha_{2}=1, k_{2}=0$ was considered in Morgül (1992) and it was shown that when $g_{2}(s)$ satisfies the above assumptions with $\gamma_{2}>0$, then the resulting closed-loop system is exponentially stable. The case $\alpha_{1}=0$ was also considered in Morgül (2001).

\section{STABILITY RESULTS}

Let the assumptions 1-3 stated above hold. Then, since the transfer function $h_{i}(s), i=1,2$, is strictly positive real it follows from the Meyer-Kalman-Yakubovich Lemma that there exist symmetric positive definite matrices $Q_{i} \in \mathbf{R}^{n_{i} \times n_{i}}$ and $P_{i} \in \mathbf{R}^{n_{i} \times n_{i}}$, a vector $q_{i} \in \mathbf{R}^{n_{i}}$ satisfying (see Slotine and Li (1991, p. 133)) :

$$
A_{i}^{T} P_{i}+P_{i} A_{i}=-q_{i} q_{i}^{T}-Q_{i}
$$




$$
P_{i} b_{i}-c_{i}=\sqrt{2\left(d_{i}-\gamma_{i}\right)} q_{i} .
$$

To analyze the system given by (1)-(3), (4)-(6), we first define the function space $\mathscr{H}$ as follows

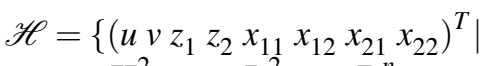

$$
\begin{aligned}
& u \in \mathbf{H}_{0}^{2}, \quad v \in \mathbf{L}^{2}, z_{i} \in \mathbf{R}^{n_{i}}, \\
& \left.x_{i 1}, x_{i 2} \in \mathbf{R}, i=1,2\right\}
\end{aligned}
$$

where the spaces $\mathbf{L}^{2}$, and $\mathbf{H}_{0}^{k}$ are defined as follows

$$
\begin{gathered}
\mathbf{L}^{2}=\left\{f:[0,1] \rightarrow \mathbf{R} \mid \int_{0}^{L} f^{2} d x<\infty\right\}, \\
\mathbf{H}^{k}=\left\{f \in \mathbf{L}^{2} \mid f^{\prime}, \ldots, f^{(k)} \in \mathbf{L}^{2}\right\}, \\
\mathbf{H}_{0}^{k}=\left\{f \in \mathbf{H}^{k} \mid f(0)=f^{\prime}(0)=0\right\} .
\end{gathered}
$$

The equations (1)-(3), (4)-(6) can be written in the following abstract form :

$$
\dot{z}=L z \quad, \quad z(0) \in \mathscr{H},
$$

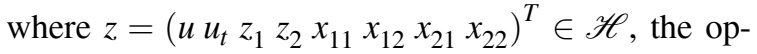
erator $L: \mathscr{H} \rightarrow \mathscr{H}$ is a linear unbounded operator defined as

$$
L\left(\begin{array}{c}
u \\
v \\
z_{1} \\
z_{2} \\
x_{11} \\
x_{12} \\
x_{21} \\
x_{22}
\end{array}\right)=\left(\begin{array}{c}
v \\
-u_{x x x x} \\
A_{1} z_{1}+b_{1} v_{x}(1) \\
A_{2} z_{2}+b_{2} v(1) \\
\omega_{1} x_{12} \\
-\omega_{1} x_{11}+v_{x}(1) \\
\omega_{2} x_{22} \\
-\omega_{2} x_{21}+v(1)
\end{array}\right) .
$$

The domain $D(L)$ of the operator $L$ is defined as

$$
\begin{aligned}
D(L)= & \left\{z \in \mathscr{H} \mid u \in \mathbf{H}_{0}^{4}, v \in \mathbf{H}_{0}^{2},\right. \\
& z_{i} \in \mathbf{R}^{n_{i}}, x_{i 1}, x_{i 2} \in \mathbf{R} ; \\
& u_{x x}(1)+\alpha_{1}\left[c_{1}^{T} z_{1}+d_{1} v_{x}(1)\right. \\
& \left.+k_{1} x_{12}\right]=0,-u_{x x x}(1)+\alpha_{2}\left[c_{2}^{T} z_{2}\right. \\
& \left.\left.+d_{2} v(1)+k_{2} x_{22}\right]=0\right\} .
\end{aligned}
$$

where $z=\left(u v z_{1} z_{2} x_{11} x_{12} x_{21} x_{22}\right)^{T}$.

Note that when $\alpha_{i}=0$ (i.e. the corresponding controller is not applied), the lines and terms corresponding to $z_{i}, x_{i 1}, x_{i 2}$ should be omitted.

Let the assumptions 1-3 hold, let $Q_{i} \in \mathbf{R}^{n_{i} \times n_{i}} P_{i} \in$ $\mathbf{R}^{n_{i} \times n_{i}}$, and $q_{i} \in \mathbf{R}^{n_{i}}$ be the solutions of (10) and (11) where $P_{i}$ is also a symmetric and positive definite matrix. In $\mathscr{H}$, we define the following "energy" innerproduct:

$$
\begin{aligned}
<y, \tilde{y}>_{E} & =\frac{1}{2} \int_{0}^{1} v \tilde{v} d x+\frac{1}{2} \int_{0}^{1} u_{x x} \tilde{u}_{x x} d x \\
& +\frac{1}{2} \sum_{i=1}^{2} \tilde{z}_{i}^{T} P_{i} z_{i} \\
& +\frac{1}{2} \sum_{i=1}^{2} k_{i}\left(x_{i 1} \tilde{x}_{i 1}+x_{i 2} \tilde{x}_{i 2}\right)
\end{aligned}
$$

where $y=\left(u v z_{1} z_{2} x_{11} x_{12} x_{21} x_{22}\right)^{T}, \tilde{y}=\left(\tilde{u} \tilde{v} \tilde{z}_{1} \tilde{z}_{2}\right.$ $\left.\begin{array}{llll}\tilde{x}_{11} & \tilde{x}_{12} & \tilde{x}_{21} & \tilde{x}_{22}\end{array}\right)^{T} \in \mathscr{H}$. It can be shown that $\mathscr{H}$, together with the energy inner-product given by (19) becomes a Hilbert space. The "energy" norm induced by (19) (for the solution $z(t)$ of (16) ) is given by :

$$
\begin{aligned}
E(t) & =\frac{1}{2} \int_{0}^{1} u_{t}^{2} d x+\frac{1}{2} \int_{0}^{1} u_{x x}^{2} d x \\
& +\frac{1}{2} \sum_{i=1}^{2} z_{i}^{T} P_{i} z_{i} \\
& +\frac{1}{2} \sum_{i=1}^{2} k_{i}\left(x_{i 1}^{2}+x_{i 2}^{2}\right) .
\end{aligned}
$$

Theorem 1 : Consider the system given by (16) with $d_{i} \geq 0$, and $k_{i} \geq 0$. The operator $L$ generates a $C_{0^{-}}$ semigroup of contractions $T(t)$ in $\mathscr{H}$, (for the terminology of semigroup theory, the reader is referred to e.g. Luo et. al. (1999)).

Proof : We use Lumer-Phillips theorem, to prove the assertion, see Luo et. al. (1999). To prove that $L$ is dissipative, we differentiate (20) with respect to time. Then by using (1)-(3), (4)-(6), integrating by parts and using (10), (11), we obtain :

$$
\begin{aligned}
\dot{E} & =\int_{0}^{1} u_{t} u_{t t} d x+\int_{0}^{1} u_{x x} u_{x x t} d x \\
& +\frac{1}{2} \sum_{i=1}^{2} z_{i}^{T}\left(A_{i}^{T} P_{i}+P_{i} A_{i}\right) z_{i} \\
& +\sum_{i=1}^{2} z_{i}^{T} P_{i} b_{i} r_{i}+\sum_{i=1}^{2} k_{i} r_{i} x_{i 2} \\
& =-u_{x x x}(1, t) u_{t}(1, t)+u_{x x}(1, t) u_{x t}(1, t) \\
& +\frac{1}{2} \sum_{i=1}^{2} z_{i}^{T}\left(A_{i}^{T} P_{i}+P_{i} A_{i}\right) z_{i} \\
& +\sum_{i=1}^{2} z_{i}^{T} P_{i} b_{i} r_{i}+\sum_{i=1}^{2} k_{i} r_{i} x_{i 2} \\
& =-\sum_{i=1}^{2} \alpha_{i} f_{i} r_{i}+\sum_{i=1}^{2} z_{i}^{T} P_{i} b_{i} r_{i} \\
& -\frac{1}{2} \sum_{i=1}^{2} z_{i}^{T} q_{i} q_{i}^{T} z_{i}-\frac{1}{2} \sum_{i=1}^{2} z_{i}^{T} Q_{i} z_{i} \\
& =-\sum_{i=1}^{2} \alpha_{i} \gamma_{i} r_{i}^{2}-\frac{1}{2} \sum_{i=1}^{2} z_{i}^{T} Q_{i} z_{i} \\
& -\frac{1}{2} \sum_{i=1}^{2}\left[\alpha_{i} \sqrt{2\left(d_{i}-\gamma_{i}\right)} r_{i}-\sum_{i=1}^{2} z_{i}^{T} q_{i}\right]
\end{aligned}
$$

where $r_{i}$ is given by (7). Since $\dot{E} \leq 0$, it follows that $L$ is dissipative, (see (19), (20), (21)). 
It can be shown that $\lambda I-L: \mathscr{H} \rightarrow \mathscr{H}$ is onto for $\lambda>0$, (see Morgül (1994) and Morgül (2001) for similar calculations). Then, it follows from the LumerPhillips theorem that $L$ generates a $C_{0}$-semigroup of contractions $T(t)$ on $\mathscr{H}$. $\square$

Note that the result stated above shows that the system under consideration is stable. Next we prove some asymptotic stability results.

Theorem 2 : Consider the system given by (16) with $d_{i} \geq 0$, and $k_{i} \geq 0$. Assume that $\alpha_{1}=\alpha_{2}=1$, (i.e. both controllers are applied).

i : If $k_{1}>0, k_{2}>0$, and either $\tau=\sqrt{\omega_{1}}$ or $\tau=\sqrt{\omega_{2}}$ are not one of the roots of the following transcendental equation :

$$
1-\cosh \tau \cos \tau=0
$$

then the semigroup $T(t)$ generated by $L$ is asymptotically stable, that is all solutions of (16) asymptotically converge to zero.

ii : If $k_{1}=0, k_{2}>0$ then the semigroup $T(t)$ generated by $L$ is asymptotically stable.

iii : If $k_{1}>0, k_{2}=0$ then the semigroup $T(t)$ generated by $L$ is asymptotically stable.

Proof : Note that by Theorem 1 , the operator $L$ generates a $C_{0}$-semigroup of contractions. To prove the assertions i-iii, we use LaSalle's invariance principle, extended to infinite dimensional systems, see Luo et. al. (1999). According to this principle, all solutions of (16) asymptotically tend to the maximal invariant subset of the following set :

$$
\mathscr{S}=\{z \in \mathscr{H} \mid \dot{E}=0\}
$$

provided that the solution trajectories for $t \geq 0$ are precompact in $\mathscr{H}$. Since the operator $L: \mathscr{H} \rightarrow \mathscr{H}$ generates a $C_{0}$-semigroup of contractions on $\mathscr{H}$ (hence the solution trajectories are bounded on $\mathscr{H}$ for $t \geq 0$ ), the precompactness of the solution trajectories are guaranteed if the operator $(\lambda I-L)^{-1}: \mathscr{H} \rightarrow \mathscr{H}$ is compact for some $\lambda>0$, see Luo et. al. (1999) To prove the last property, we first show that $L^{-1}$ exists and is a compact operator on $\mathscr{H}$. To see this, let $q=\left(f h r_{1} r_{2} r_{11} r_{12} r_{21} r_{22}\right)^{T} \in \mathscr{H}$ be given. We want to solve the equation $L z=q$ for $z$, where $z=$ $\left(u v z_{1} z_{2} x_{11} x_{12} x_{21} x_{22}\right)^{T} \in D(L)$. After straightforward calculations, the required $z \in D(L)$ can be found uniquely. It follows that $L^{-1}$ exists and maps $\mathscr{H}$ into $\mathbf{H}^{4} \times \mathbf{H}^{2} \times \mathbf{R}^{n} \times \mathbf{R} \times \mathbf{R}$. Since $q \in \mathscr{H}$ it follows that $f \in \mathbf{H}_{0}^{2}$, see (12). Hence, if $\|q\|$ is bounded in $\mathscr{H}$, it follows easily that that $f(1)$ is bounded as well. Therefore $L^{-1}$ maps the bounded sets of $\mathscr{H}$ into the bounded sets of $\mathbf{H}^{4} \times \mathbf{H}^{2} \times \mathbf{R}^{n} \times \mathbf{R} \times \mathbf{R}$. Since the embedding of the latter into $\mathscr{H}$ is compact, see Tanabe (1979, p. 14), it follows that $L^{-1}$ is a compact operator. This also proves that the spectrum of $L$ consists entirely of isolated eigenvalues, and that for any $\lambda$ in the resolvent set of $L$, the operator $(\lambda I-L)^{-1}: \mathscr{H} \rightarrow \mathscr{H}$ is a compact operator, Kato (1980, p. 187). Furthermore, our argument above shows that $\lambda=0$ is not an eigenvalue of $L$. Since the operator $L$ generates a $C_{0}$-semigroup of contractions on $\mathscr{H}$, by the argument given above it follows that the solutions trajectories of (16) are precompact in $\mathscr{H}$ for $t \geq 0$, hence by LaSalle's invariance principle, the solutions asymptotically tend to the maximal invariant subset of $\mathscr{S}$ (see (23)). Hence, to prove that all solutions of (16) asymptotically tend to the zero solution, it suffices to show that $\mathscr{S}$ contains only the zero solution, which is a typical procedure in the application of LaSalle's invariance principle.

To prove that $\mathscr{S}$ contains only the zero solution, we set $\dot{E}=0$ in (21), which results in $z_{i}=0, i=1,2$. This implies that $\dot{z}_{i}=0$, hence by using (4) and (6) we obtain $r_{i}(t)=0, f_{i}(t)=k_{i} x_{i 2}, i=1,2$. Hence, all solutions of (16) in $\mathscr{S}$ satisfy the following equation $(i=1,2)$

$$
\begin{gathered}
u_{t t}+u_{x x x x}=0, \\
\dot{x}_{i 1}=\omega_{i} x_{i 2}, \dot{x}_{i 2}=-\omega_{i} x_{i 1}, \\
u(0, t)=0, u_{x}(0, t)=0, \\
u_{x t}(1, t)=0, u_{t}(1, t)=0, \\
-u_{x x}(1, t)=k_{1} x_{12}, u_{x x x}(1, t)=k_{2} x_{22}
\end{gathered}
$$

The solution $x_{i 2}$ of (25) can be written as :

$$
x_{i 2}=a_{i} \cos \left(\omega_{i} t+\theta_{i}\right) \text {, }
$$

where $a_{i}$ and $\theta_{i}$ are arbitrary constants.

Since the boundary conditions in (26), (27) are separable, the solution $u$ of (24) can be found by using separation of variables, see Meirovitch (1967). That is, the solution of (24), (26), and (27) assumes the following form : $u(x, t)=A(t) B(x)$ where the functions $A: \mathbf{R}_{+} \rightarrow \mathbf{R}$ and $B:[0,1] \rightarrow \mathbf{R}$ are differentiable functions to be determined from the boundary conditions. We distinguish the following cases :

a : $\dot{A} \equiv 0$. In this case, the solutions of (24) is $u(x, t)=$ $c_{0}+c_{1} x+c_{2} x^{2}+c_{3} x^{3}$. From (26) and (27), it follows that $c_{i}=0, i=0, \ldots, 4$, and $a_{i}=0$. Hence, the only possible solution is $u(x, t) \equiv 0$.

b : $\dot{A} \neq 0$. In this case, the solution of (24) and (26) is in the following form :

$$
\begin{gathered}
A(t)=c \cos (\omega t+\theta), \\
B(x)=c_{3}(\cosh \tau x-\cos \tau x) \\
+c_{4}(\sinh \tau x-\sin \tau x),
\end{gathered}
$$

where $\tau=\sqrt{\omega}$, and $c, \theta, c_{3}, c_{4}$ are arbitrary constants. By using (31) in (27), it can be shown that in order to have a nontrivial solution, $\tau$ should satisfy (22). By using (31) and (29) in (28), it can be shown that 
to have a nontrivial solution, both $\tau=\sqrt{\omega_{1}}$ and $\tau=$ $\sqrt{\omega_{2}}$ must be a root of (22). It can be easily shown that if this condition fails, then the only possible solution is the trivial solution. Hence, by LaSalle's invariance principle, we conlude that the solutions of (16) asymptotically tend to the zero solution.

ii, iii can be proven similarly. Note that in the case ii, in addition to the boundary conditions (26) and (27), we will have $u_{x x}(1, t)=0$, whereas for the case iii, we will have $u_{x x x}(1, t)=0$ as well. By using separation of variables, it can easily be shown that the only solution of (24) together with these boundary conditions is the zero solution. Hence, again by LaSalle's invariance theorem, we obtain the stated asymptotic stability result.

The case $\alpha_{1}=0, \alpha_{2}=1$ (i.e. only the boundary control force is applied) has been considered in Morgül (2001). Next we give a result for the remaining case $\alpha_{1}=1, \alpha_{2}=0$.

Theorem 3 : Consider the system given by (16) with $d_{i} \geq 0$, and $k_{i} \geq 0$. Assume that $\alpha_{1}=1, \alpha_{2}=0$, (i.e. only the boundary control torque is applied). If $k_{1}>0$ and $\tau=\sqrt{\omega_{1}}$ is not one of the roots of the following transcendental equation

$$
\sinh \tau \cos \tau+\cosh \tau \sin \tau=0
$$

then the semigroup $T(t)$ generated by $L$ is asymptotically stable, that is all solutions of (16) asymptotically converge to zero.

Proof : The proof of this fact is similar to that of Theorem 2, and hence is omitted here.

\section{DISTURBANCE REJECTION}

In this section we show the effect of the proposed control law given by (4)-(6) on the solutions of the system given by (1)-(3), when the output of the controller is corrupted by a disturbance $n_{i}(t)$, that is (6) has the following form :

$$
f_{i}(t)=c_{i}^{T} z_{i}+d_{i} r_{i}+k_{i} x_{i 2}+n_{i}(t)
$$

or equivalently we have the following :

$$
\hat{f}_{i}(s)=g_{i}(s) \hat{r}_{i}(s)+\hat{n}_{i}(s)
$$

for $i=1,2$, where a hat denotes the Laplace transform of the corresponding variable $g_{i}(s)$ is given by (8).

To find the transfer function from $n_{i}$ to $r_{i}$, first we need to find the transfer function from $f_{i}$ to $r_{i}$. By taking the Laplace transform of (1)-(3) and using zero initial conditions, after some straightforward calculations we obtain the following : $(i=1,2)$

$$
\hat{r}_{i}(s)=-h_{i 1}(s) \hat{f}_{1}(s)-h_{i 2}(s) \hat{f}_{2}(s)
$$

where $h_{i j}(s)$ are appropriate functions, which are not given here due to space limitations. After straightforward calculations, from (34), (35) we obtain $(i=1,2)$ :

$$
\hat{r}_{i}(s)=\tilde{h}_{i 1}(s) \hat{n}_{1}(s)+\tilde{h}_{i 2}(s) \hat{n}_{2}(s),
$$

where $\tilde{h}_{i j}(s)$ are the closed-loop transfer functions.

From (36) we can also derive a procedure to design $g_{i}(s)$ if we know the structure of $n_{i}(t)$. For example if $n_{i}(t)$ has a band-limited frequency spectrum, (i.e. has frequency components in an interval of frequencies $\left.\left[\Omega_{1}, \Omega_{2}\right]\right)$, then we can choose $g_{i}(s)$ to minimize $\left|\tilde{h}_{i j}(j \omega)\right|$ for $\omega \in\left[\Omega_{1}, \Omega_{2}\right]$, for $i, j=1,2$. Note that to ensure the stability of the closed-loop system, $g_{i}(s)$ should be a positive real function as well, (see (8)). As a simple example, assume that $n_{1}(t)=0, n_{2}(t)=$ $a \cos \omega_{0}(t)$. Then we may choose $\alpha_{1}=0, \alpha_{2}=1$ (i.e. only boundary control force is applied), and $g_{2}(s)$ in the form (8) with $\omega_{2}=\omega_{0}$. Provided that the assumptions 1-3 are satisfied and that $j \omega_{0}$ is not a zero of $h_{22}(s)$, the closed-loop system is asymptotically stable. Moreover, if $k_{2}>0$, then we have $\tilde{h}_{22}\left(j \omega_{0}\right)=0$. From the above discussions we may conclude that this eliminates the effect of the disturbance at the output $u_{t}(1, t)$, see also Morgül (1998), Morgül (2001).

\section{CONCLUSION}

In this paper, we considered a linear time invariant system which is represented by one-dimensional EulerBernoulli beam equation in a bounded domain. We assumed that the system is clamped at one end and the boundary control force and torque inputs are applied at the other end. For this system, we proposed finite dimensional dynamic boundary controllers to generate the input force and torque. This introduces extra degrees of freedom in designing controllers which could be exploited in solving a variety of control problems, such as disturbance rejection, pole assignment, etc., while maintaining stability. The transfer function of the controllers are proper rational functions of the complex variable $s$, and may contain poles on the imaginary axis, provided that the residues corresponding to these poles are nonnegative; the rest of the transfer function is required to be strictly positive real function. We then proved that the closed-loop system is stable in general and asymptotically stable under certain conditions. These conditions depend on the location of the imaginary axis poles. We also discussed the case where the output of the controller is corrupted by a disturbance. We showed that, if the frequency spectrum of the controller is known, then by choosing the controller appropriately we may obtain better disturbance rejection.

\section{REFERENCES}

Balas, M. J. (1978), "Modal Control of Certain Flexible Dynamical Systems," SIAM J. of Contr. and Opt. vol.16, pp.450-462. 
Chen, G. (1979), "Energy Decay Estimates and Exact Boundary Value Controllability for the Wave Equation in a Bounded Domain," J. Math. Pures. Appl., vol.58, pp.249-273.

Chen,G., M. C. Delfour, A. M. Krall and G. Payre, (1987), "Modelling, Stabilization and Control of Serially Connected Beams," SIAM J. of Contr. and Opt., vol.25, pp.526-546.

Kato, T. (1980), Perturbation Theory for Linear Operators, 2nd. ed. New York : Springer Verlag.

Luo, Z. H., Guo, B. Z., and Morgül, Ö., (1999), Stability and Stabilization of Infinite Dimensional Systems with Applications, Springer-Verlag, series in Communications and Control, London.

Meirovitch, L. (1967), Analytical Methods in Vibration, New York, Mac Millan.

Morgül, Ö. (1990) " Control and Stabilization of a Flexible Beam Attached to a Rigid Body," Int. J. Contr., vol. 51, pp. 11-33.

Morgül, Ö. (1991), "Orientation and Stabilization of a Flexible Beam Attached to a Rigid Body : Planar Motion," IEEE Trans. on Auto. Contr., vol 36, No. 8, pp. 953-963.

Morgül, Ö. (1992), "Dynamic Boundary Control of a Euler-Bernoulli Beam," IEEE Transactions on Auto. Contr., vol. 37, no. 5, pp. 639-642.

Morgül, Ö. (1994), "Dynamic Boundary Control of the Wave Equation," Automatica, vol. 30, no. 11, pp. 1785-1792.

Morgül, Ö. (1998), "Stabilization and Disturbance Rejection for the Wave Equation," IEEE Transactions on Auto. Contr., vol. 43, no. 1, pp. 89-95.

Morgül, Ö. (2001), "Stabilization and Disturbance Rejection for the Beam Equation," accepted for publication in IEEE Transactions on Auto. Contr.

Pazy, A. (1983), Semigroups of Linear Operators and Applications to Partial Differential Equations, New York, Springer-Verlag.

Tanabe, H., (1979), Equations of Evolution, Pitman.

Slotine, J. J. E., and Li, W., (1991), Applied Nonlinear Control, Englewood Cliffs, New Jersey, Prentice-Hall. 